\title{
Thermal Expansion of Crystal Lattice of Isotactic Poly(4-methyl-1-pentene)
}

\author{
Tetsuya Tanigami, Kazuo Yamaura, Shuji Matsuzawa, \\ and Keizo MIYASAKA* \\ Department of Chemistry, Faculty of Textile Science and Technology, Shinshu University, \\ Ueda-shi, Nagano 386, Japan \\ * Department of Textile and Polymeric Materials, Tokyo Institute of Technology, \\ Ookayama, Meguro-ku, Tokyo 152, Japan
}

(Received June 15, 1985)

\begin{abstract}
The thermal expansion of the crystal lattice of the isotactic poly(4-methyl-1pentene) (P4M1P) was studied by X-ray diffraction in the temperature range between $-170^{\circ} \mathrm{C}$ and $180^{\circ} \mathrm{C}$. The range was divided into nine small regions of different expansion coefficients. Various kinds of molecular motions in some of these regions and transition points are discussed in relation to the anisotropy of the expansion and the dynamic mechanical properties of the sample. Interestingly, in two lower temperature-ranges between $-126^{\circ} \mathrm{C}$ and $-72^{\circ} \mathrm{C}$, and between $-45^{\circ} \mathrm{C}$ and $-13^{\circ} \mathrm{C}$ the expansion coefficients abruptly increased along the $a$-axis whereas decreased along the $c$-axis. The thermal expansibility of the chain is also discussed in relation to the anisotropy of the elastic modulus of the crystal.
\end{abstract}

KEY WORDS Poly(4-methyl-1-pentene) / Thermal Expansion / Crystal Lattice / Molecular Motion / Dynamic Mechanical Property / Density / XRay Diffraction /

Molecular motion in poly(4-methyl-1pentene) (P4M1P) was investigated in the 1960 's. ${ }^{1-15}$ Takayanagi et al. successfully elucidated the dynamic mechanical behavior by using its single crystals. ${ }^{9-11}$ On the other hand, Rånby et al. undertook the first systematic investigation on the dependency of the dynamic mechanical properties on the crystal structural variation with temperature. ${ }^{1,2}$ However, he measured the crystal expansion in the range only above $20^{\circ} \mathrm{C}$. Therefore, some mechanical relaxation processes below room temperature have not yet been compared with the molecular motion in the crystal phase. Thus, in this paper, we report on the thermal expansion of the crystal lattice in the range between $-170^{\circ} \mathrm{C}$ and $180^{\circ} \mathrm{C}$. Such a technique of X-ray diffraction has already been used for polyethylene (PE) crystals. $^{16,17}$

The following two expectations motivated us to do this work: one is that the molecular motion in the crystal phase would not be negligible, owing to the extraordinarily small density of the crystal phase and the other that the expansion along the chain axis would be responsive to the conformational variation with temperature, owing to the small elastic modulus along it.

\section{EXPERIMENTAL}

\section{Sample Preparation}

The P4M1P used in this study was a product of Mitsui Petrochemical Co., Ltd. (TPX $\left.{ }^{\circledR}\right)$. Films with about $0.5 \mathrm{~mm}$ thickness were prepared by pressing polymer pellets at $260^{\circ} \mathrm{C}$ and quenching in ice water. One of these films was then annealed at $200^{\circ} \mathrm{C}$ for $20 \mathrm{~min}$ in a silicone oil bath. This unoriented specimen was used for the dynamic mechanical measurement. The 
others were drawn uniaxially to five times the original length using a manually operated device in boiling water, and subsequently annealed at $200^{\circ} \mathrm{C}$ for $20 \mathrm{~min}$. These specimens were used for X-ray diffraction. The crystallinity estimated by the usual X-ray diffraction method of these two types of specimens was $33 \%$.

\section{Measurements}

Throughout this work, Ni-filtered $\mathrm{Cu}-K_{\alpha}$ radiation was used for $\mathrm{X}$-ray diffraction. The $\mathrm{X}$-ray diffraction photographs were taken by a flat plate camera. The expansion of the crystal lattice was measured by use of meridional 007 reflection and equatorial 200, 220, and 420 reflections. These diffraction profiles were recorded by a Rigaku-Denki scintillation counter at various temperatures. The temperature was controlled by blowing nitrogen gas evaporated from liquid nitrogen. It was necessary to use an inert gas such as nitrogen in order to avoid oxidation at high temperature and condensation of water at low temperature.

Dynamic mechanical properties were measured as a function of temperature by using a Toyo-Seiki Reolograph. The frequency was $10 \mathrm{~Hz}$ and the heating rate was $3^{\circ} \mathrm{C} \mathrm{min.}{ }^{-1}$

\section{RESULTS}

\section{(1) X-ray Diffraction Pattern}

$X$-ray diffraction patterns of the sample were taken at $-80,25$, and $160^{\circ} \mathrm{C}$. No change of the pattern was observed, as already reported. $^{2}$ Namely, it was confirmed that the crystal lattice is tetragonal and the conformation of the chain is a $7 / 2$ helix throughout the temperature range of measurement.

\section{(2) Thermal Expansion along the a-Axis}

Figure 1 shows the crystal lattice parameter $a$ as a function of temperature, which was derived from the spacings of (200), (220), and (420) planes. These plots were obtained by repeatings two times the heating and cooling

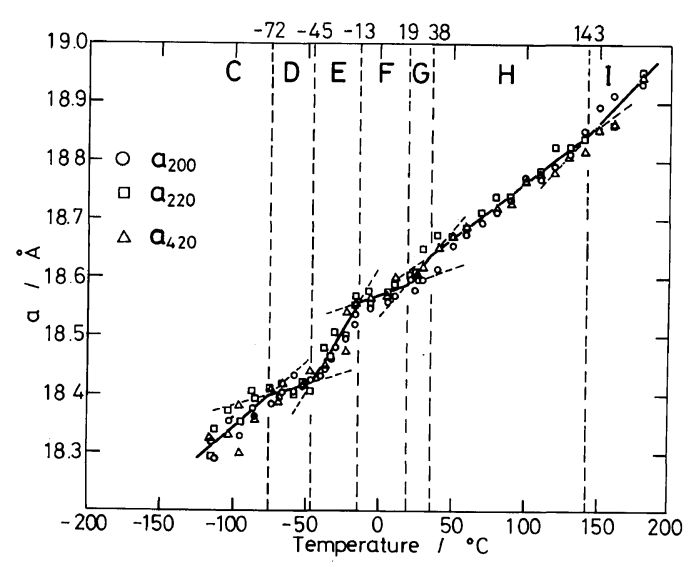

Figure 1. Change in the lattice parameter $a$ with temperature. The three symbols in the Figure show the diffractions from which the parameter was calculated.

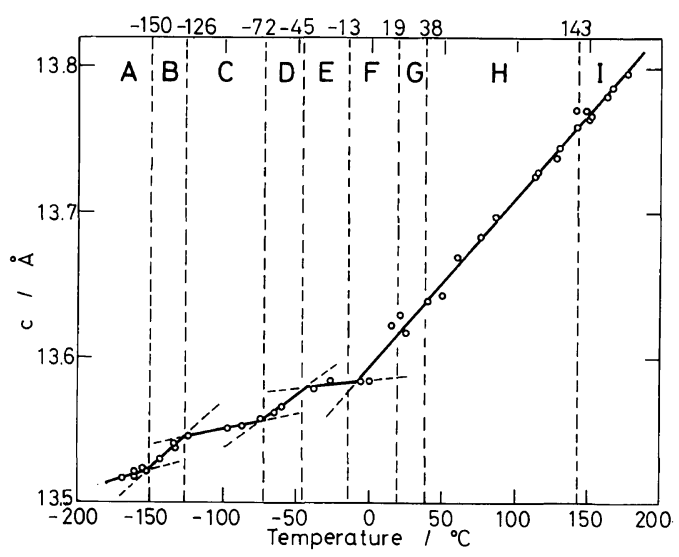

Figure 2. Change in the lattice parameter $c$ with temperature.

processes. Assuming that the expansion was partially linear with temperature, the curve could be divided into seven smaller regions by six bending points. They were termed regions $\mathbf{C}, \mathbf{D}, \cdots, \mathbf{I}$ in the order of ascending temperature. The most significant observation was that, at regions $\mathbf{C}, \mathbf{E}$, and $\mathbf{G}$, the parameter abruptly increased.

\section{(3) Thermal Expansion along the c-Axis}

Figure 2 shows the temperature dependency of the lattice parameter $c$ calculated from the spacings of the (007) plane. The curve could be 
divided into six straight lines. The divisions in Figure 1 could be also applied to this case, provided that regions $\mathbf{A}$ and $\mathbf{B}$ were added to it and regions $\mathbf{F}, \mathbf{G}, \mathbf{H}$, and $\mathbf{I}$ were taken as one region. The expansion was noticeably restrained at regions $\mathbf{C}$ and $\mathbf{E}$. It was noted that, in these regions, the expansion along the $a$ axis increased.

\section{(4) Dynamic Mechanical Property}

Figure 3 shows the tensile storage modulus $E^{\prime}$ and the loss modulus $E^{\prime \prime}$ as a function of temperature. Five relaxations were observed on the $E^{\prime \prime}$ curve: $\beta_{\mathrm{sc}}, \beta_{\mathrm{a}}, \alpha_{\mathrm{a}}, \beta_{\mathrm{c}}$, and $\alpha_{\mathrm{c}}$ in the order of ascending temperature. After a series of measurements performed on various kinds of samples with different crystallinity. Takayanagi et al. reached the following conclusion $:^{9-11} \beta_{\mathrm{sc}}$-relaxation is due to the side chain activity associated with the frozen amorphous chains $\left(\beta_{\mathrm{sc}}-\mathrm{A}\right)$ and with the defect region within the crystal phase $\left(\beta_{\mathrm{sc}}-\mathrm{C}\right) ; \beta_{\mathrm{a}}$-relaxation is due to the local rotational mode of the main chain around its chain axis which is associated with the bulky side chain (isobutyl group); $\alpha_{a^{-}}$ relaxation is due to the micro Brownian motion of the amorphous segments (primary relaxation); $\beta_{\mathrm{c}}$-relaxation is due to the intermolecular friction and the torsional rigidity of the molecular axis which are activated by the thermal expansion in the crystal phase and $\alpha_{c}-$ relaxation is due to the translational vibration along the molecular axis in the crystal phase.

The drawing effects of the sample on the temperature range of the relaxation is considered to be negligible from Choy et al.'s report. ${ }^{15}$ Thus, the ranges observed for the unoriented sample were compared with the crystal expansion for the oriented sample, as shown in Figure 3, to give the following three points:

(a) The transition point of $\mathrm{T} 1$ from regions $\mathbf{A}$ to $\mathbf{B}$, and that of $\mathrm{T} 2$ from $\mathbf{H}$ to $\mathbf{I}$ correspond to the $\beta_{\mathrm{sc}}$ - and $\alpha_{\mathrm{c}}$-peaks, respectively.

(b) Regions $\mathbf{E}$ and $\mathbf{G}$ overlap with $\beta_{\mathrm{a}}$ - and $\alpha_{\mathrm{a}}$ relaxations, respectively.

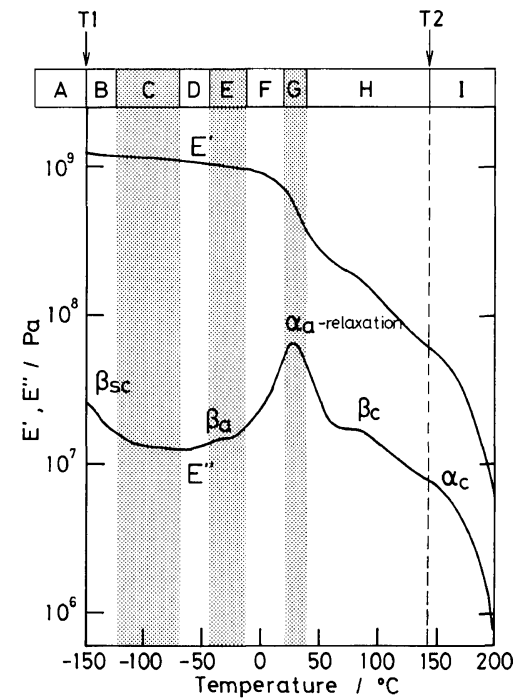

Figure 3. Tensile storage modulus $E^{\prime}$ and loss modulus $E^{\prime \prime}$ versus temperature for the unoriented specimen.

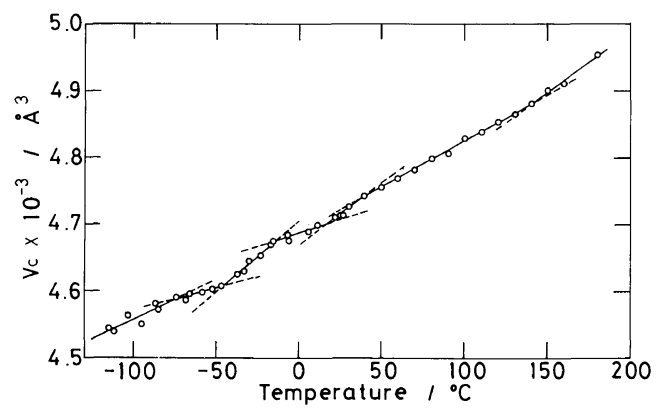

Figure 4. Variation of volume of the unit cell $\left(V_{c}\right)$ with temperature which was calculated from the data in Figures 1 and 2.

(c) Corresponding relaxation and expansion regions are not found for region $\mathbf{C}$ and $\beta_{\mathrm{c}}$ relaxation, respectively.

\section{(5) Volumetric Expansion of the Crystal Unit Cell}

Figure 4 shows the volume of the crystal unit cell as a function of temperature, which was calculated from the data in Figures 1 and 2. The greater part of its expansion depends on the expansion along the $a$-axis.

As Figure 5 shows, the density of the crystal phase was calculated as a function of tempera- 


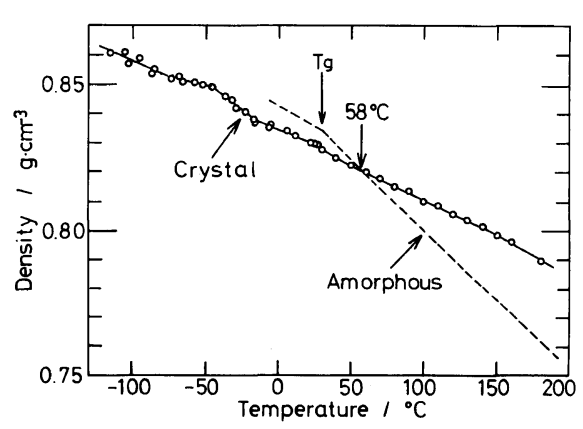

Figure 5. Densities of the crystal and amorphous phases as a function of temperature. The former was calculated from the data in Figure 4, and the latter was after Griffith et al.'s. ${ }^{1}$

ture from the data in Figure 4, assuming four molecular chains to be involved in the unit cell. The amorphous density shown in the figure was after Griffith et al.'s. ${ }^{1}$ It was clearly shown that the crystal density is less than the amorphous one below room temperature, and that the cross point of the two densities is $58^{\circ} \mathrm{C}$.

\section{DISCUSSION}

\section{(1) Anisotropy of the Crystal Expansion}

Figure 6 shows the thermal expansion coefficients along the $a$-axis $\left(\alpha_{\mathrm{a}}\right)$ and $c$-axis $\left(\alpha_{\mathrm{c}}\right)$ as a function of temperature. The coefficient of thermal expansion is a two-rank tensor. In orthogonal lattices, the principal axes of the expansion coincide with those of their crystal unit cell. In this case, the tensor is expressed as

$$
\left[\begin{array}{lll}
\alpha_{1}^{\circ} & 0 & 0 \\
0 & \alpha_{1}^{\circ} & 0 \\
0 & 0 & \alpha_{3}^{\circ}
\end{array}\right]
$$

The thermal expansion coefficient in an optional direction in the $a c$-plane can be expressed as a function of the azimuth angle $(\theta)$ made by $a$-axis and temperature $(T)$ :

$\alpha(T, \theta)=\alpha_{1}^{\circ}(T) \cos ^{2} \theta+\alpha_{3}^{\circ}(T) \sin ^{2} \theta$

The patterns of the anisotropy are shown for the various temperature ranges (Figure 7), which are calculated by applying the data in

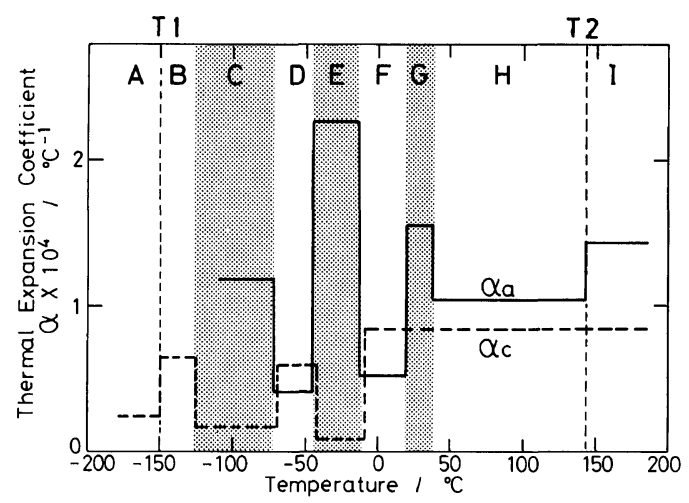

Figure 6. Changes in the thermal expansion coefficients with temperature.

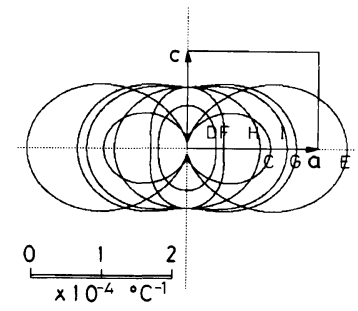

Figure 7. Anisotropic patterns of the thermal expansion coefficient in the $a c$-plane for the various temperature ranges.

Figure 6 to eq 1. Two types of expansion anisotropy can be distinguished from these patterns, the group of regions $\mathbf{D}, \mathbf{F}, \mathbf{H}$, and $\mathbf{I}$, and that of regions $\mathbf{C}, \mathbf{E}$, and $\mathbf{G}$. In the former group, the coefficient increases stepwise in the order of the temperature range in all the directions. In the latter group, on the other hand, positive and negative projecting changes occur in the directions of $a$ and $c$-axes, respectively.

In general, the expansion can be affected either by conformational change of the chain itself, or by activated intermolecular interaction. The former is caused by changes in bond length, bond angle, or rotational angle around the bond axis. Thus it can be detected with greater correspondence in the dimensional change in $c$-axis. The latter effects largely the lateral expansion with respect to the chain axis. From these points of view, the special 
expansions of P4M1P were characterized on the two transition points and in the two types of transition regions as follows. However, it was noted that some speculation still cannot be avoided from the discussion on the expansion along $a$-axis, since the equatorial diffraction profile unfortunately could not be obtained below $-120^{\circ} \mathrm{C}$ by our apparatus.

(i) Transition Point T1 from Regions $\mathbf{A}$ to $\mathbf{B}$. The expansion along $c$-axis was conspicuous at about $-150^{\circ} \mathrm{C}$ (T1), where the $\beta_{\text {sc }}$-relaxation peak was located. Interestingly, also in some polymers of planar zigzag chains such as nylon $6(\alpha \text {-form })^{18}$ and ethylene-tetrafluoroethylene copolymer, ${ }^{19}$ the occurrence of the (negative) expansion along the chain axis was observed with " $\gamma$-relaxation" which was attributed to local motion of amorphous chains. The negative expansion is attributed to an extended conformation which is easily shrunk by a rotational motion around the bond axis. In the $7 / 2$ helix, the rotational motion should cause positive expansion.

(ii) Transition Regions $\mathbf{C}$ and $\mathbf{E}$. In both regions $\mathbf{C}$ and $\mathbf{E}, \alpha_{\mathrm{a}}$ abruptly increases accompanying with an abrupt decrease of $\alpha_{c}$. This linkage of the two expansions indicates the existence of a certain kind of small but abrupt conformational change. The effect of this change is not so big as to make the total $\alpha_{c}$ negative. Furthermore, it would be caused by a relaxation mode characteristic of each region. The twisting motion of the side chain has been observed at $-60^{\circ} \mathrm{C}$ by ESR and NMR measurements ( $\beta_{\mathrm{a}}$-relaxation) and attributed to that in the amorphous phase rather than the crystal one. ${ }^{9}$ However, the crystal expansion at these rigions supports the attribution of the crystal phase to this relaxation, which may be more effective in this polymer than in the other crystalline polymers because of its small crystal density.

(iii) Transition Region G. In region G, $\alpha_{\mathrm{a}}$ abruptly increases without any change of $\alpha_{c}$. This may also be related to $\alpha_{\mathrm{a}}$-relaxation originarily attributed to the motion of the amorphous chain. It is difficult to determine whether the motive motion of the expansion is intramolecular or intermolecular. Probably, both the motions may cause this expansion.

(iv) Transition Point T2 from Regions $\mathbf{H}$ to $\mathbf{I}$. At the transition point of $\mathrm{T} 2\left(143^{\circ} \mathrm{C}\right)$, only $\alpha_{\mathrm{a}}$ slightly increases. The rotational motion around the axis of the crystal molecule would cause the intermolecular expansion, when it is activated by $\alpha_{\mathrm{c}}$-relaxation.

From the above discussion, it seems that the transitions at $\mathrm{T} 1$ and $\mathrm{T} 2$ are of second-order and the transition at regions $\mathbf{C}$ and $\mathbf{E}$ are of

Table I. Comparison of expansion coefficients with the elastic modulus of crystals for P4M1P and polyethylene

\begin{tabular}{|c|c|c|c|c|c|c|}
\hline \multirow{2}{*}{ Polymer } & \multicolumn{3}{|c|}{ Expansion coefficient $\times 10^{4} / \mathrm{K}^{-1}$} & \multicolumn{3}{|c|}{ Elastic modulus/GPa } \\
\hline & $\alpha_{\mathrm{a}}$ & $\alpha_{\mathrm{b}}$ & $\alpha_{c}$ & $E_{\mathrm{a}}$ & $E_{\mathrm{b}}$ & $E_{\mathrm{c}}$ \\
\hline Poly(4-methyl-1-pentene) & \multicolumn{2}{|c|}{$\begin{array}{c}1.04^{\mathrm{a}} \\
\left(\alpha_{\mathrm{a}}=\alpha_{\mathrm{b}}\right)\end{array}$} & $0.85^{\mathrm{a}}$ & \multicolumn{2}{|c|}{$\begin{array}{c}2.9^{\mathrm{b}} \\
\left(E_{\mathrm{a}}=E_{\mathrm{b}}\right)\end{array}$} & $2.9^{\mathrm{b}}$ \\
\hline Polyethylene & $2.21^{\mathrm{c}}$ & $0.38^{\mathrm{c}}$ & $-0.035^{\mathrm{d}}$ & $3.2^{\mathrm{e}}$ & $3.9^{\mathrm{e}}$ & $235^{\mathrm{e}}$ \\
\hline
\end{tabular}

a Our data from region $\mathrm{H}$.

b Ref 21.

c Ref 22.

${ }^{d}$ Ref 23.

e Ref 24.

Subscripts a, b, and c attached to $\alpha$ and $E$ denote the unit cell axes. 


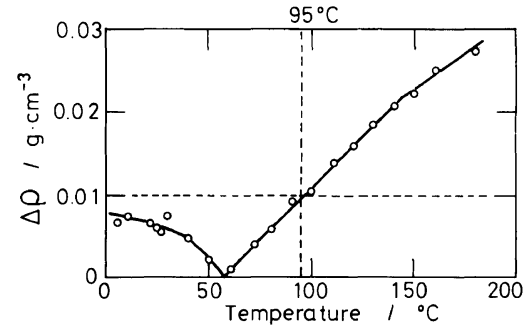

Figure 8. Absolute value of the density difference between the crystal and amorphous phases as a function of temperature.

first-order.

\section{(2) Comparison of the Thermal Expansion} Coefficient with the Elastic Modulus

The thermal expansion is compared with the elastic modulus for PE and P4M1P crystals in Table I. The anisotropy between the parallel and perpendicular directions to the chain axis is comparable to that between the thermal expansion coefficient and the elastic modulus. The observed value of the expansion coefficient seems to be almost proportional to the reciprocal of that of the modulus. The small anisotropy of P4M1P could be due to the small elastic modulus in the chain axis. Furthermore, the loosely spiraled chain structure with the large side chain introduces a large cross-sectional area of the molecule, extraordinarily small density of the crystal phase, and this small modulus in the chain axis.

\section{(3) Density Difference between the Crystal and Amorphous phases}

Figure 8 shows the absolute values of the density difference $(\Delta \rho)$ as a function of temperature, calculated from Figure 5. As $\Delta \rho$ is very small at room temperature, the intensity of small-angle X-ray scattering (SAXS) could not be observed. However, it was shown that heating is effective to increase the SAXS intensity. ${ }^{20}$ The lowest temperature above which the intensity could be observed was $95^{\circ} \mathrm{C}$. Thus the lowest density difference to be detected by SAXS may be about $0.01 \mathrm{~g} \mathrm{~cm}^{-3}$ as shown in
Figure 8, if a conventional SAXS apparatus is used.

\section{REFERENCES}

1. J. H. Griffith and B. G. Rånby, J. Polym. Sci., 44, 369 (1960).

2. B. G. Rånby, K. S. Chan, and H. Brumberger, $J$. Polym. Sci., 58, 545 (1962).

3. K. S. Chan, B. G. Rånby, H. Brumberger, and A. Odajima, J. Polym. Sci., 61, 171 (1962).

4. B. G. Rånby, Kobunshi, 13, 197 (1964).

5. A. E. Woodward, J. A. Sauer, and R. A. Wall, $J$. Polym. Sci., 50, 117 (1960).

6. A. E. Woodward, A. Odajima, and J. A. Sauer, $J$. Phys. Chem., 65, 1384 (1961).

7. B. I. Hunt, J. G. Powles, and A. E. Woodward, Polymer, 5, 323 (1964).

8. J. M. Crissman, J. A. Sauer, and A. E. Woodward, J. Polym. Sci., A, 2, 5075 (1964).

9. N. Kusumoto, K. Shirano, and M. Takayanagi, Kogyo Kagaku Zasshi, 68, 1553 (1965).

10. Y. Tanda, K. Imada, and M. Takayanagi, Kogyo Kagaku Zasshi, 69, 1971 (1966).

11. M. Takayanagi and N. Kawasaki, J. Macromol. Sci., Phys., B1, 741 (1967).

12. S. Yamada and Y. Konakahara, Kobunshi Kagaku, 23, 521 (1966).

13. F. Sakaguchi, W. Tsuji, and R. Kitamaru, Kobunshi Kagaku, 24, 318 (1967).

14. K. Shimizu, O. Yano, and Y. Wada, J. Polym. Sci., Polym. Phys. Ed., 13, 1959 (1975).

15. C. L. Choy, W. K. Luk, and F. C. Chen, Polymer, 22, 543 (1981).

16. M. Takayanagi, T. Aramaki, M. Yoshino, and K. Hoashi, J. Polym. Sci., 46, 148 (1960).

17. M. Takayanagi, Memoirs Fac. Eng. Kyushu Univ., 23, 41 (1963).

18. K. Miyasaka, T. Isomoto, H. Koganeya, K. Uehara, and K. Ishikawa, J. Polym. Sci., Polym. Phys. Ed., 18, 1047 (1980).

19. T. Tanigami, K. Yamaura, S. Matsuzawa, M. Ishikawa, K. Mizoguchi, and K. Miyasaka, to be published.

20. T. Tanigami and K. Miyasaka, J. Polym. Sci., Polym. Phys. Ed., 19, 1865 (1981).

21. K. Kaji, I. Sakurada, K. Nakamae, T. Shintaku, and E. Shikata, Bull. Inst. Chem. Res. Kyoto Univ., 52, 308 (1974).

22. P. R. Swan, J. Polym. Sci., 56, 403 (1962).

23. Y. Kobayashi and A. Keller, Polymer, 11, 114 (1970).

24. I. Sakurada and K. Kaji, J. Polym. Sci., C, 31, 57 (1970); Preprints, Kobe Meeting of the Society of Polymer Science, Jpn., (1975), p 56. 